\title{
GERENCIAMENTO DE MATERIAIS E CUSTOS HOSPITALARES EM UTI NEONATAL
}

\author{
Lauren Auer Lopes ${ }^{1}$, Ana Maria Dyniewicz² ${ }^{2}$ Luísa Canestraro Kalinowski ${ }^{3}$
}

\begin{abstract}
RESUMO: Esta pesquisa objetivou quantificar e qualificar o uso indevido de materiais de consumo em uma Unidade de Terapia Intensiva Neonatal de um hospital universitário da cidade de Curitiba/PR. Foi realizada observação não participante na unidade no período de 17 de março a 2 de abril de 2009, em todos os turnos de trabalho, totalizando 100 horas. Os resultados revelaram o emprego indevido de materiais de consumo; uso de materiais de baixa qualidade; uso irracional; e improvisos, além de falhas no manuseio por profissionais. A pesquisa mostrou o volume e o valor de gastos impróprios na unidade, com alguns destaques para cateteres venosos periféricos, gazes, sondas nasogástricas, ampolas de glicose, cabos de oxímetros, entre outros. Sugere-se que a unidade melhore a gestão, por meio de auditoria de materiais e custos hospitalares, com olhar crítico e consciente sobre racionalização de uso de materiais.
\end{abstract}

PALAVRAS-CHAVE: Custos hospitalares; Armazenamento de materiais e provisões; Recursos materiais em saúde.

\section{MATERIALS MANAGEMENT AND HOSPITAL COSTS IN NEONATAL ICU}

\begin{abstract}
This study aimed to quantify and qualify the improper use of consumables in a Neonatal Intensive Care Unit of a university hospital in Curitiba / PR. Non-participant observation was conducted in the unit during the period of March 17 to April 2, 2009, on all shifts, totaling 100 hours. The results revealed the improper use of consumables, the use of low quality materials, irrational use, and improvisation, as well as failures in handling the materials by professionals. The survey showed the volume and value of improper spending in the unit, with some highlights for peripheral venous catheters, gauzes, nasogastric tubes, ampoules of glucose, oximeters cables, among others. It is suggested that the unit must improve the management of materials and hospital costs through auditing, critically and consciously about the rational use of materials.
\end{abstract}

KEYWORDS: Hospital costs; Storage of materials and supplies; Material resources in health.

\section{GERENCIAMENTO DE MATERIALES Y COSTOS HOSPITALARIOS EN UTI NEONATAL}

RESUMEN: Esta investigación objetivó cuantificar y cualificar el uso indebido de materiales de consumo en una Unidad de Terapia Intensiva Neonatal de un hospital universitario de la ciudad de Curitiba/PR. Fue realizada observación no participante en la unidad en el periodo de 17 de marzo a 2 de abril de 2009, en todos los turnos de trabajo, totalizando 100 horas. Los resultados revelaron el empleo indebido de materiales de consumo; uso de materiales de baja calidad; uso irracional; e improvisados, además de fallas en el manoseo por profesionales. La investigación mostró el volumen y el valor de gastos impropios en la unidad, con algunos destaques para catéteres venosos periféricos, gasas, sondas nasogástricas, ampollas de glucosa, cables de oximetros, entre otros. Se sugiere que la unidad mejore la gestión, por medio de auditoría de materiales y costos hospitalarios, con visión crítica y consciente sobre racionalización de uso de materiales.

DESCRIPTORES: Costos hospitalarios; Almacenamiento de materiales y provisiones; Recursos materiales en salud.

${ }^{1}$ Enfermeira da Prefeitura Municipal de Curitiba-PR. Especialista em Auditoria e Gestão em Saúde.

${ }^{2}$ Enfermeira. Doutora em Enfermagem. Membro do Grupo de Pesquisas em Políticas, Gestão e Práticas em Saúde-GPPGPS, do Departamento de Enfermagem da Universidade Federal do Paraná-UFPR

${ }^{3}$ Enfermeira. Mestranda do Programa de Pós-Graduação em Enfermagem da UFPR. Bolsista CAPES.

Autor correspondente:

Ana Maria Dyniewicz

Universidade Federal do Paraná

Rua Padre Anchieta, 1965 - 80730-000 - Curitiba-PR

Recebido: 23/10/09

E-mail: anadyni@yahoo.com.br

Aprovado: 04/03/10

Cogitare Enferm. 2010 Abr/Jun; 15(2):278-85 


\section{INTRODUÇÃO}

As crescentes medidas de redução de gastos dos governos observadas nos últimos anos em todas as esferas foram sentidas, e muito, no setor da saúde. Assim sendo, a redução de custos tornou-se imprescindível ${ }^{(1)}$. Essa questão torna-se vital para as empresas e, por conseguinte, para os hospitais, sendo que a busca pela redução de gastos passa a ser uma constante. Ou seja, a implementação de sistemas de gerenciamento de custos é fundamental, principalmente quando se objetiva conter os gastos, sem perder a qualidade do serviço prestado $^{(2)}$.

Concomitantemente, um dos problemas do planejamento em saúde no Brasil é a questão da qualidade da assistência à saúde, o que pode dificultar a previsão de recursos materiais, assim como o estabelecimento da relação entre oferta de serviços e demandas de saúde. A identificação dessas dificuldades permite redirecionar recursos e atividades aos grupos de usuários mais problemáticos ou de maior risco, o que certamente levaria a uma redução dos recursos despendidos ${ }^{(3)}$. Há, contudo, que se considerar a forma como a instituição está estruturada e organizada internamente, ou seja, se as condições de trabalho que oferece são melhores ou piores, com mais ou menos possibilidades de desempenho intelectual e manual, acesso a recursos, tipos de relacionamentos hierárquicos, níveis de satisfação e desgaste, entre outros ${ }^{(4)}$.

Neste alinhamento reflexivo, a administração de recursos materiais dentro dos hospitais tem recebido maior atenção, pois junto com os recursos humanos, os recursos financeiros formam a base de sustentação do hospital, sendo este último um de seus elementos principais. Trata-se de um ciclo contínuo de operações correlatas e interdependentes que são previsão, aquisição, transporte, recebimento, armazenamento, distribuição, conservação, venda de excedentes e análise de controle de inventários, o que assegura ao hospital o reabastecimento racional dos materiais necessários à manutenção de seu ciclo operacional ${ }^{(5)}$.

Cabe aos profissionais de saúde, em especial ao enfermeiro, gerenciar e administrar recursos materiais, que são os que mais elevam os custos hospitalares, depois dos recursos humanos. É de suma importância que os enfermeiros participem do processo de seleção e compra de materiais, pois têm como avaliar se a quantidade e a qualidade do produto condizem com as necessidades da clientela e de sua equipe, que deve ser treinada constantemente, a fim de evitar desperdícios e, por extensão, o aumento do custo hospitalar por uso indevido desses materiais ${ }^{(6)}$.

Convém destacar, ainda, que os recursos materiais nas organizações de saúde englobam diversos fatores, tais como a proporção que a despesa com materiais representa para a instituição; a grande variedade de materiais; a complexidade de tratamentos e, por consequência, o ônus nos custos. Além disso, os custos hospitalares têm aumentado consideravelmente devido à complexidade de tratamentos e procedimentos, exigindo materiais mais modernos e caros para sua execução ${ }^{(7)}$.

Para auxiliar nesse sentido, uma das tendências para o gerenciamento de serviços de enfermagem é a inclusão de conhecimentos de auditoria e desenvolvimento de habilidades sobre custos como mais uma ferramenta a ser utilizada nos processos decisórios, sendo importante o envolvimento de toda equipe de enfermagem na criação de um programa de educação continuada e desenvolvimento da auditoria ${ }^{(7)}$.

Diante disso, esta pesquisa objetivou analisar o uso de recursos materiais em Unidade de Terapia Intensiva Neonatal (UTI Neonatal) de um hospital universitário. Especificamente, busca quanti-qualificar o uso indevido de materiais de consumo.

\section{METODOLOGIA}

O presente estudo, de abordagem quantitativa realizado por meio da observação não participante, foi conduzido em UTI Neonatal de hospital universitário de Curitiba, Estado do Paraná.

A unidade em questão tem 14 leitos e nela trabalham 100 técnicos em enfermagem, 66 médicos e 33 enfermeiros; pertence a uma maternidade a qual conta com 50 leitos e atende em parceria com o Estado e Prefeitura Municipal. A demanda é alta, 1.700 consultas de pronto-atendimento e cerca de 320 partos por mês, o que corresponde a, aproximadamente, um terço dos partos realizados pelo Sistema Único de Saúde (SUS) na capital paranaense.

A coleta dos dados foi realizada por observação não participante e ocorreu no período de 17 de março a dois de abril de 2009, nos quatro turnos de trabalho: manhã, tarde, noite I e noite II, por três horas diárias, totalizando 100 horas de observação, no período de 3 semanas.

Para a coleta de dados foi elaborada uma listagem de materiais de maior consumo na unidade e, a 
cada período de observação, foram realizadas anotações sobre as características na utilização desses materiais. Os dados foram transcritos para planilha do programa Excel, submetidos à estatística descritiva e apresentados na forma de quadros, favorecendo sua análise e discussão.

A população do estudo foi composta por enfermeiros, médicos e profissionais de nível médio, de todos os turnos da unidade.

Foi solicitada previamente a autorização da direção do referido hospital para realização da pesquisa. Após parecer positivo, o projeto foi encaminhado e aprovado sob n. 000120/2009 no Comitê de Ética da Universidade Tuiuti do Paraná. O estudo foi realizado segundo as diretrizes e normas regulamentadoras de pesquisa envolvendo seres humanos, conforme Resolução 196/96 do Conselho Nacional de Saúde, que trata desse tipo de pesquisa, sendo que cada participante assinou o Termo de Consentimento Livre e Esclarecido, com garantia de seu anonimato e sigilo.

No período de observação, os pacientes internados foram classificados, de acordo com o grau de complexidade, em críticos e não-críticos.

\section{RESULTADOS E DISCUSSÃO}

Os resultados e as discussões a seguir tratam das 100 horas de observações em uma UTI Neonatal, cujos dados foram categorizados e estão apresentados abaixo. Primeiramente, há uma caracterização do grau de complexidade dos pacientes atendidos e apresentada na tabela 1.

Tabela 1 - Grau de complexidade de pacientes internados em UTI Neonatal. Curitiba, 2009.

\begin{tabular}{|c|c|c|c|c|}
\hline $\begin{array}{l}\text { Grau de } \\
\text { Complexidade }\end{array}$ & $1^{\text {a Semana }}$ & $2^{\mathrm{a}}$ Semana & $3^{a}$ Semana & Total \\
\hline & $\mathrm{n}$ & $\mathrm{n}$ & $\mathrm{n}$ & $\mathrm{n}$ \\
\hline Críticos & 23 & 15 & 15 & 53 \\
\hline Não-Críticos & 121 & 119 & 39 & 279 \\
\hline Total & 144 & 134 & 54 & 332 \\
\hline
\end{tabular}

Durante o período de observação foram levantados os itens caracterizados como uso indevido de materiais de consumo, que foram categorizados em: materiais de baixa qualidade; emprego habitual inadequado; improviso; e inabilidade profissional. Os detalhes sobre essa categorização estão apresentados na tabela a seguir:
Tabela 2 - Uso indevido de materiais em UTI Neonatal por baixa qualidade do produto. Curitiba, 2009.

\begin{tabular}{lc}
\hline Material & Quantidade/ Unidade \\
\hline 1. Cateter venoso periférico & 23 \\
2. Caneta esferográfica & 17 \\
3. Curativo Filme Transparente & 14 \\
4. Cateter umbilical & 12 \\
5. Extensor de equipo & 06 \\
6. Torneirinha 3 vias & 06 \\
7. Papel toalha & 06 \\
8. Sonda de aspiração & 05 \\
9. Kit CPAP Neo & 03 \\
\hline Total & 91 \\
\hline
\end{tabular}

Neste estudo, a classificação - produto de baixa qualidade - se relaciona àqueles que, ao proceder uma atividade, o profissional identifica que não possui a qualidade necessária para a devida utilização. Durante o período foi observado que 91 produtos tinham inadequações e, por essa razão, foi preciso utilizar um número maior desses para realizar os procedimentos necessários. Um exemplo foi o uso de 23 cateteres venosos periféricos, com defeitos, requerendo a necessidade de mais punções, provocando maior sofrimento à criança, além de aumento dos riscos de infecção e gasto excessivo.

A punção venosa periférica é procedimento invasivo e complexo em UTI Neonatal e exige, portanto, destreza e competência tecnicocientífica. É um procedimento muito utilizado por equipes de enfermagem, com riscos ao paciente e passível de acidente biológico para o profissional. Sendo assim, e embora haja no mercado uma variedade de produtos, cabe ao enfermeiro, nas dimensões gerenciais e educacionais próprias de seu processo de trabalho, a tomada de decisões e apoio ao uso de tecnologias eficientes à qualidade do cuidado.

Essa situação possibilita refletir sobre a ineficiência de um processo gerencial em saúde, como reflexos de irresponsabilidade administrativa diante dos recursos do SUS, referente às despesas de custos de procedimentos terapêuticos.

Outra situação de baixa qualidade de materiais foi relativa aos extensores de equipo, que por seis vezes apresentaram vazamento, impossibilitando seu uso. Igualmente, o papel toalha extremamente fino, sendo necessário o uso de pelo menos seis folhas para secar as mãos. Também foram encontradas canetas esfero- 
gráficas que deixavam de escrever, embora com tinta suficiente, e kits de Pressão Positiva Contínua nas Vias Aéreas (CPAP) deformados. Nesse aspecto, ficou a dúvida, se foi devido à baixa qualidade do produto, ou se a deformação deveu-se ao reprocessamento do artigo.

Em 12 situações observou-se que os cateteres umbilicais apresentaram dois problemas: alguns não eram radiopacos, o que dificultava a identificação de localização anatômica correta depois de inserido, e outros não apresentavam as marcas de graduação que facilitam a inserção. As torneirinhas de três vias, utilizadas nas extremidades de cateteres umbilicais, apresentaram o mesmo problema dos extensores de equipo: houve vazamento ou não se encaixavam à extremidade do cateter, em seis situações. As válvulas das sondas de aspiração não funcionaram em cinco situações.

Em Neonatologia, o Curativo Filme Transparente deve ser o de menor tamanho disponível no mercado, ou seja, compatível com a clientela pediátrica. Contudo, por 14 vezes o material foi perdido por inadequação.

A descrição acima, sobre materiais inaproveitáveis, permite analisar que a estrutura formal das organizações hospitalares depende de negociações que seus agentes estabeleçam entre si, em diferentes áreas de trabalho, bem como dos processos de interação para manter em funcionamento as atividades diárias da instituição e a organização do processo de cuidado ${ }^{(8)}$.

Assim, em setores de prestação de serviços em que há preocupação com a implantação ou implementação de gestão de qualidade, é importante a descentralização de decisões para que o sistema de comunicação seja eficiente, sem distanciamento das relações interfuncionais e a individualização no desempenho das atividades. Isso será possível se houver conhecimento dos papéis organizacionais, da missão das unidades administrativas, as comunicações intensivas e o trabalho cooperativo, sistêmico e totalmente integrado ${ }^{(9)}$.

A criação de uma cultura com essas características é obtida quando voltada para o cliente interno, como elemento essencial para que o serviço e suas unidades funcionem e, tragam satisfação e conhecimento das expectativas e necessidades dos clientes. Nessa perspectiva, o desempenho das áreas de suporte, tal como compra de materiais e suprimentos, subentende administração solidária nas relações que vão desde fornecedores externos, passando pelas áreas de suporte, até alcançar as áreas de linha-de-frente, que atendem aos clientes internos. Dessa maneira, justifica-se estudar mais profundamente o impacto que a integração e sensibilização para ações conjuntas entre as áreas fim e meio proporcionam na melhoria contínua da qualidade dos serviços ofertados aos clientes ${ }^{(9)}$.

No que diz respeito a custos, foram gastos com materiais de baixa qualidade $\mathrm{R} \$ 630,65$ durante o período de estudo. Esse valor corresponderia à compra, por exemplo, de um sensor e cabo para oxímetro e um kit de CPAP para o setor.

A tabela 3 trata do uso irracional de materiais por profissionais na UTI Neonatal pesquisada. Nomeiase uso irracional o gasto impróprio ou sem proveito, inaderente, próprio de uma ação de desperdício.

Tabela 3 - Relação de materiais por uso irracional em UTI Neonatal. Curitiba, 2009.

\begin{tabular}{lc}
\hline Material & Quantidade/Unidade \\
\hline 1. Gaze & 102 situações \\
2. Fita crepe & 28 situações \\
3. Luva de procedimento & 26 unidades \\
4. Filme plástico transparente & 18 situações \\
5. Sonda gástrica & 14 unidades \\
6. Saco plástico & 07 unidades \\
7. Frasco água destilada $1000 \mathrm{ml}$ & 06 unidades \\
8. Oxigênio & 4 dias de vazamento \\
9. Cabo oxímetro & 03 unidades \\
\hline Total & 208 \\
\hline
\end{tabular}

Houve, em 102 ocasiões, a utilização de gazes sem necessidade, quer seja para desinfecção de incubadoras, berços, bancadas e equipamentos, quer seja para auxiliar na oferta de leite e/ou dieta ao recémnascido, sendo que a unidade dispõe de compressas próprias para tais fins.

As luvas de procedimento foram utilizadas de maneira inadequada em 26 situações. Com elas, os profissionais fizeram uma "chupeta" com um dedo da luva e algodão embebido em solução glicosada, oferecida ao recém-nascido. Esse procedimento é habitual na unidade para controle da dor e para acalmar o bebê. Pesquisas mostram que parece haver sinergismo entre o uso de chupetas (sucção não-nutritiva) e a glicose no alívio da dor, logo é possível recomendar o emprego clínico de solução glicosada por via oral, cerca de um a dois minutos antes de pequenos procedimentos, como punções capilares, venosas ou arteriais ${ }^{(10)}$. Com essa recomendação, busca-se alternativa para o conforto da criança de maneira segura e econômica, o que dispensaria o uso de luvas aqui relatado. 
Como mostra a tabela 3, sondas gástricas foram usadas em 14 situações para amarrar e pendurar as pranchetas dos registros de enfermagem nas incubadoras e berços. Nesse caso, poderia ser utilizado outro material de menor custo, a ser retirado em cada alta hospitalar. Frascos de água destilada de um litro foram usados para repor líquido na câmara de aquecedor de CPAP, campânula e respirador, e frasco da Pressão Positiva Expiratório Final (PEEP). No entanto, como são necessários $250 \mathrm{ml}$ ou $125 \mathrm{ml}$, evitarse-ia o desperdício de, pelo menos, $500 \mathrm{ml}$.

Ainda, foi observada a utilização de 18 pedaços, de aproximadamente $1.40 \mathrm{~cm}$, de filme plástico polietileno para proteger equipamentos e mobiliários do setor, mas esse material tem por finalidade: envolver o berço para manter a temperatura; aquecer o bebê; manter aquecido o recém-nato transferido do centro obstétrico para a UTI Neonatal; envolver o berço sob calor radiante. Na unidade, também foi observado o uso do filme com esses propósitos, que são reafirmados na literatura para evitar as perdas de calor por convecção e radiação, inclusive nas laterais dos berços em calor radiante ${ }^{(11)}$.

Na observação, também foi constatado o uso de saco plástico para envolver estetoscópio e termômetro que são colocados dentro das incubadoras ou berços preparados para admissão dos recém-nascidos. Partimos do pressuposto que o material, devidamente higienizado, não carece de invólucros nos leitos dos pacientes.

Durante quatro dias de observação na unidade constatou-se vazamento contínuo de oxigênio de uma das válvulas fixadas na parede. Infelizmente, não foi possível avaliar quantos litros foram desperdiçados por minuto e nem qual foi o custo para a instituição. Em relação aos cabos e sensores de oxímetros observou-se que, por três vezes, houve descuido no manuseio ao fazer a desinfecção, e os cabos se quebraram. Nesta pesquisa, o uso irracional de materiais e equipamentos totalizou R\$1.565,49.

A respeito da observação de materiais perdidos por uso inadequado, destaca-se que a Neonatologia é uma especialidade que requer conhecimentos específicos da equipe de enfermagem, sendo necessária a aquisição de um acervo de habilidades e atitudes que permitam ao enfermeiro e à equipe, progressiva segurança na execução de técnicas, com responsabilidade e eficiência.

Contudo, a contínua busca pela eficiência tecnicocientífica requer uso racional de recursos, sem aquisição de arranjos ou improvisações indevidas de materiais e equipamentos que possam provocar práticas inconsequentes em serviços de saúde. Há, também, que considerar que cabe ao enfermeiro quebrar essas possíveis resistências de rever utilizações incorretas, em especial devido aos riscos aos pacientes e alto custo em UTI Neonatal.

Por outro lado, é importante destacar que a UTI Neonatal, como parte do sistema macroestrutural dos serviços de saúde, tem pacientes com maior ameaça à vida, principalmente aqueles que se encontram em estado crítico. Por conseguinte, o atendimento exige rapidez e habilidade técnica no desempenho da ação para evitar ocorrências iatrogênicas. No entanto, há falhas nas condições de trabalho e provisão inadequada de materiais e equipamentos, tornando o processo de cuidar frustrante. E mesmo assim os profissionais acabam fazendo o melhor que podem, mas, ainda assim, isso pode culminar em prejuízo para a qualidade do cuidar $^{(12)}$.

A tabela 4 mostra situações de improviso feitas por profissionais da UTI Neonatal pesquisada:

Tabela 4 - Relação de materiais com gasto excessivo, por improviso, em UTI Neonatal. Curitiba, 2009.

\begin{tabular}{lc}
\hline Material & Quantidade/Unidade \\
\hline 1. Micropore & 43 situações \\
2. Esparadrapo & 08 situações \\
3. Papel alumínio & 06 situações \\
4. Circuito de respirador & 04 unidades \\
\hline Total & 61 \\
\hline
\end{tabular}

Como mostra a tabela acima o micropore, em 43 situações, e o esparadrapo, em 8 ocasiões, foram usados para fechar as roupas dos recém-nascidos ou fraldas descartáveis desprovidas de fitas adesivas. Observou-se, também, o uso do esparadrapo para adaptar as traqueias do CPAP às conexões e fixar seringas às incubadoras.

Compreende-se a necessidade de tais materiais na prática em neonatologia, contudo, pela observação, seria interessante que o seu uso fosse racional, ou seja, que houvesse pelos profissionais maior compreensão sobre o problema crescente de custos hospitalares e de sua repercussão social.

O papel alumínio foi utilizado em seis situações para proteger os equipos usados para administrar a Nutrição Parenteral Total, que é fotossensível. Embora a literatura aponte que os equipos devam ser protegidos, não há especificações sobre como fazê-lo. Sabe- 
se, contudo, que a fotossensibilidade está relacionada à embalagem, à estabilidade depois da diluição e ao tempo de validade dos medicamentos após o preparo - acondicionados em embalagens especiais - e as instruções para o manuseio desses medicamentos devem ser rigorosamente seguidas ${ }^{(13)}$.

Houve falta de peças dos circuitos de respiradores em quatro situações, o que ocasionou a abertura de outros dois circuitos para montar um completo. Esses materiais são de alto custo e essenciais ao serviço.

As práticas de improviso acontecem nos serviços de saúde, seja para adequar uma técnica com o que há disponível para uso, ou porque já fazem parte da rotina.

“As carências de material permanente e/ou consumo e de recurso humano exigem que se crie, que se faça de um nada um tudo para que não falte o cuidado de enfermagem a ser prestado"(14:747).

No entanto, meios improvisados não são totalmente eficazes e podem causar danos à saúde, embora

\begin{abstract}
"a improvisação originada das práticas para dar conta das técnicas seja considerada por muito tempo com pouca cientificidade e sem valor de criação, mas sempre foi um dos pontos fortes do cuidar em Enfermagem”(14:747).
\end{abstract}

Observou-se, nessa categoria, um gasto de R $\$$ 3.401,23 decorrente de improvisação. Com essa realidade, destaca-se que cabe ao gestor da unidade adequar equipamentos, recursos materiais e processos de trabalho, além de manter a equipe continuamente informada sobre os valores de tais materiais, como mais uma forma de conscientização sobre racionalização de custos.

Convém considerar, contudo, que a incorporação de novas tecnologias em saúde não substitui trabalho por capital, ao contrário, aumenta a necessidade de mão-de-obra cada vez mais complexa e sofisticada. Porém, nas atuais condições de trabalho a que estão sujeitos os profissionais de saúde, falta de salários condizentes, escassez de mão-de-obra qualificada, custos sociais elevados, ocorre baixa produtividade e atenção inadequada à saúde ${ }^{(15)}$.

Por outro lado, há responsabilidade, por parte das instituições de saúde, em investir na educação das equipes para oferecer assistência de qualidade consoante a custos aceitáveis. A busca pela competência técnico-científica acontece por meio de formação e atualização constantes do seu capital humano, tornando-se a linha mestra de uma equipe de saúde crítica e consciente do seu papel profissional ${ }^{(16)}$.
Na tabela 5 estão elencados medicamentos utilizados na unidade pesquisada, com manuseio inadvertido, pois requeriam melhor preparo técnico e científico por parte dos profissionais.

Tabela 5 - Medicamentos manipulados incorretamente em UTI Neonatal. Curitiba, 2009.

\begin{tabular}{|c|c|}
\hline Material & Quantidade/Unidade \\
\hline 1. Glicose $50 \%$ & 77 \\
\hline 2. Dimorf ${ }^{\circledR}$ & 06 \\
\hline 3. Gentamicina ${ }^{\circledR}$ & 05 \\
\hline 4. Damina & 04 \\
\hline 5. Dobutamina & 04 \\
\hline 6. Fentany $\mathrm{l}^{\circledR}$ & 03 \\
\hline 7. Fenobarbital ${ }^{\circledR}$ & 03 \\
\hline 8. Aminofilina ${ }^{\circledR}$ & 03 \\
\hline 9. Surfactante ${ }^{\circledR}$ & 02 \\
\hline Total & 107 \\
\hline
\end{tabular}

Nos resultados sobre o uso incorreto de medicamentos destaca-se a necessidade de melhor preparo técnico-científico dos profissionais que atuam na UTI Neonatal, a fim de se tornar rotina o uso consciente dos recursos disponíveis no setor, como por exemplo em relação à estabilidade das medicações após diluição, otimizando seu uso, como no caso das ampolas de gentamicina, Dimorf ${ }^{\circledR}$ e Fentanyl ${ }^{\circledR}$. Em outro estudo, também constatou-se fato semelhante em relação ao uso de medicações, principalmente as injetáveis ${ }^{(17)}$.

Em pesquisa bibliográfica sobre o assunto, a ocorrência de erros na assistência ao recém-nascido decorre da complexidade dos procedimentos e do peso muito baixo dos neonatos, além da contínua introdução de novas tecnologias. Os autores relatam que os problemas mais comuns na administração de medicamentos são erro decorrente do cálculo de doses muito pequenas; velocidade de infusão; conversão de miligramas para microgramas; administração incorreta; e erros na interpretação da prescrição. Os efeitos adversos podem levar à infecção, apneia, distúrbio de perfusão periférica, alterações eletrolíticas e ácido-básicas, acarretando intervenções desnecessárias. As taxas de potenciais eventos adversos em recém-natos é maior que em outros pacientes e, na maioria dos casos, os erros são considerados passíveis de prevenção( ${ }^{(18)}$.

Em outro aspecto, para a racionalização de recursos seria necessário que a unidade pesquisada adotasse doses unitárias de medicamentos, nos casos 
de fenobarbital, dopamina, dobutamina e aminofilina, os quais não possuem estabilidade após abertos ou diluídos. Se assim fosse feito, haveria a redução de gastos no valor de $\mathrm{R} \$ 1.611,58$.

Em relação a custos hospitalares há crescentes dificuldades no desenvolvimento da prática da equipe de saúde pela utilização de procedimentos e tratamentos complexos, tal como ocorre em unidades críticas. Essas exigem estrutura física adequada, pessoal capacitado e materiais cada vez mais modernos que, por um lado, facilitam o desenvolvimento de atividades e, por outro, trazem grande dependência entre essa prática e os equipamentos clínicos adotados. O nível de complexidade tecnológica se refletirá em tarefas a serem executadas por pessoal capacitado e estratégias institucionais de otimização de tempo e de recursos, aumentando, consequentemente, a produção de serviços, reduzindo custos e possibilitando a eficiência e eficácia dos procedimentos, o que resulta em qualidade da assistência ${ }^{(19)}$.

A crescente elevação dos custos na assistência à saúde trouxe aos profissionais a necessidade de aquisição deste conhecimento, para racionalização no processo de alocação de recursos, equilíbrio entre recursos financeiros e otimização de resultados. Por outro lado, o crescimento exponencial desses custos está diretamente relacionado a uma série de fatores, tais como o emprego de novas tecnologias; o crescimento da demanda; a escassez de mão-de-obra qualificada, acarretando baixa produtividade; a má gestão das organizações devido à incapacidade administrativa dos profissionais de saúde; a nãoimplantação de sistemas de controle; os desperdícios na cadeia produtiva, entre outros ${ }^{(17)}$.

\section{CONCLUSÃO}

O estudo mostrou a quantidade e a qualidade de materiais, equipamentos e medicamentos em uso indevido em uma unidade crítica. Vale lembrar que a instituição é um hospital que possui convênio exclusivo com o SUS, e como tal, merece melhor auditoria de materiais. Ainda, o serviço pesquisado adota o modelo de compra direta, mantendo elevado estoque de insumos.

Sugere-se que o gestor da unidade, em conjunto com o setor de compras, organize um portfólio qualificado de fornecedores de produtos, além programas internos de conscientização sobre o uso racional dos materiais.
Nas 100 horas de obervação para a coleta de dados foram constatados gastos por uso indevido num total de R\$ 7.208,95, o que é de grande impacto no orçamento hospitalar. Embora as limitações dos dados tenham impedido um estudo mais meticuloso sobre o tema, bem como a incorporação de outras situações particularmente importantes a serem observadas, espera-se que estes resultados sirvam de estímulo a novas investigações e aprofundamentos sobre o tema.

Os resultados evidenciaram a necessidade da equipe de saúde da UTI Neonatal em estudo promover momentos para reflexão e discussão, nos aspectos técnicos e operacionais, acerca da otimização do uso de materiais. Finaliza-se destacando que o gestor deve estar constantemente com um olhar crítico sobre os processos de trabalho, sensibilizando equipes de saúde quanto ao uso e manuseio de recursos materiais.

\section{REFERÊNCIAS}

1. Maeheler AE, Cerreta PS, Cassanego P. Aplicação do método de criticidade de materiais em estoques hospitalares. In: XXIV Encontro Nacional de Engenharia de Produção; 2004 Nov. Florianópolis, Brasil. Florianópolis: ENEGEP; 2004.

2. Lourenço KG, Castilho V. Classificação ABC dos materiais: uma ferramenta gerencial de custos em enfermagem. Rev Bras Enferm. 2006;59(1):52-5.

3. Costal CMA, Guimarães RM. Considerações sobre a administração de recursos materiais em um hospital universitário. Rev Enferm UERJ. 2004;12(2):205-10.

4. Oliniski SR, Lacerda MR. As diferentes faces do ambiente de trabalho em saúde. Cogitare Enferm. 2004;9(2):43-52.

5. Barbosa EA, Azevedo LG, Villar AM. Gestão de estoques em materiais de uso hospitalar: análise de modelo utilizado num hospital universitário público. In: XXIV Encontro Nacional de Engenharia de Produção; 2004 Nov. Florianópolis, Brasil. Florianópolis: ENEGEP; 2004.

6. Kurcgant P. Administração em enfermagem. São Paulo: EPU; 1991.

7. Padilha MAS. Importância da educação continuada na redução de custos hospitalares. In: XVI CIC pesquisa e responsabilidade ambiental; 2007 Dez. Pelotas, Brasil. Pelotas: UFPel; 2007. 
8. Collet N, Rocha SMM. Criança hospitalizada: mãe e enfermagem compartilhando o cuidado. Rev Latino-Am Enfermagem. 2004;12(2):191-7.

9. Ibiapina FLP. Avaliando a qualidade das áreas de suporte em uma instituição de saúde [trabalho de conclusão de curso]. Fortaleza (CE): Universidade Gama Filho; 2005.

10. Silva YP, Gomez RS, Máximo TA, Silva ACS. Sedação e analgesia em neonatologia. Rev Bras Anestesiol. [Internet] 2007;57(5) [acesso em 19 abr 2010]. Disponível: http://www.scielo.br/pdf/rba/v57n5/en_13.pdf

11. Scochi CGS, Gaiva MAM, Silva MHA, Riul MJS. Termorregulação: assistência hospitalar ao recémnascido pré-termo. Acta Paul Enferm. 2002;15(1):72-8.

12. Leite MA, Vila VSC. Dificuldades vivenciadas pela equipe multiprofissional na unidade de terapia intensiva. Rev Latino-Am Enfermagem. 2005;13(2):145-50.

13. Telles Filho PCP, Cassiani SHB. Medication administration: acquisition of knowledge and abilities required for a group of nurses. Rev Latino-Am Enfermagem. 2004;12(3):533-40.

14. Coelho MJ. Maneiras de cuidar em enfermagem. Rev Bras Enferm. 2006;59(6):745-51.

15. Francisco IMF, Castilho V. A enfermagem e o gerenciamento de custos. Rev Esc Enferm. USP. 2002;36(3):240-4.

16. Torres MM, Andrade D, Santos CB. Punção venosa periférica: avaliação de desempenho dos profissionais de enfermagem. Rev Latino-Am Enfermagem. 2005;13(3):299-304.

17. Aranha GTC, Vieira RW. Estudo de um dos indicadores do custo da qualidade: o desperdício. Rev Adm Saúde. 2004;6(23):43-55.

18. Carvalho M, Vieira AA. Erro médico em pacientes hospitalizados. J Pediatr. 2002;78(4): 261-8.

19. Madureira CR, Veiga K, Sant'ana AFM. Gerenciamento de tecnologia em terapia intensiva. Rev Latino-Am Enfermagem. 2000;8(6):68-75. 